\title{
Thresholds for Linear Optics Quantum Computing with Photon Loss at the Detectors
}

\author{
Marcus Silva, ${ }^{1,2}$ Martin Rötteler, ${ }^{3}$ and Christof Zalka ${ }^{1,2}$ \\ ${ }^{I}$ Institute for Quantum Computing, University of Waterloo, \\ 200 University Ave. W, Waterloo, Ontario, N2L 3G1, Canada \\ ${ }^{2}$ Department of Physics, University of Waterloo, 200 University Ave. W, Waterloo, Ontario, N2L 3G1, Canada \\ ${ }^{3}$ NEC Laboratories America, Inc., 4 Independence Way, Princeton, NJ 08540, U.S.A.
}

(Dated: November 10, 2018)

\begin{abstract}
We calculate the error threshold for the linear optics quantum computing proposal by Knill, Laflamme and Milburn [Nature 409, pp. 46-52 (2001)] under an error model where photon detectors have efficiency $<100 \%$ but all other components - such as single photon sources, beam splitters and phase shifters - are perfect and introduce no errors. We make use of the fact that the error model induced by the lossy hardware is that of an erasure channel, i.e., the error locations are always known. Using a method based on a Markov chain description of the error correction procedure, our calculations show that, with the 7 qubit CSS quantum code, the gate error threshold for fault tolerant quantum computation is bounded below by a value between $1.78 \%$ and $11.5 \%$ depending on the construction of the entangling gates.
\end{abstract}

PACS numbers: 03.67.Lx

Keywords: error correction, threshold, linear optics, quantum computing, Markov chains

\section{INTRODUCTION}

In Refs. 1, 2 it was demonstrated how a quantum computer could be built using only single photon sources, passive linear optics elements, and photon detectors. Quantum computing proposals that use photons to encode information are particularly interesting because of practical applications to quantum communication over optical fibers, and the natural resilience of photons to decoherence. This proposal is also a conceptual departure from other quantum computing proposals because it requires post-selection of states in order to overcome the limitations imposed by the choice of physical resources - namely, the fact that one cannot make photons interact using passive linear optics elements. Moreover, even when considering ideal hardware (i.e. lossless and infinitely precise linear optics elements, $100 \%$ efficient detectors) one must use error correction codes to make the implementation efficient [2]. However, photon detectors are necessary for the qubit measurements used in post-selection and error correction, and good photon detectors are notoriously hard to build. In this paper, we investigate the maximum error rate that a linear optics quantum computer, as proposed in Ref. 1, can sustain, assuming that the only source of hardware imperfections is the finite photon loss at the photon detectors.

The paper is organized as follows. In Section describe the construction of a probabilistic gate as given in Ref. 1 and emphasize the description of the error model which arises naturally from considering those gates. In Section III we describe an error model based on the same gate constructions, but assuming that the single photon detectors have less than perfect efficiency. In Section IV the error correction code and the circuits used for error correction are described, along with constraints for fault-tolerance. Finally, in Section $\nabla I$ the recursion relations for the error rates at different level of concatenated encoding are given under worst case assumptions, along with a brief description of how they were calculated, and the threshold values are stated.

Throughout the paper we will use the Pauli matrices $\mathbf{X}=$ $\left(\begin{array}{ll}0 & 1 \\ 1 & 0\end{array}\right), \mathbf{Y}=\left(\begin{array}{rr}0 & -i \\ i & 0\end{array}\right), \mathbf{Z}=\left(\begin{array}{rr}1 & 0 \\ 0 & -1\end{array}\right)$ along with the identity matrix $\mathbb{1}$.

\section{IDEAL HARDWARE ERROR MODEL}

In the efficient linear optics quantum computing proposal put forward by Knill, Laflamme and Milburn [1], qubits are encoded as a single photon in one of two optical modes, that is, $|0\rangle$ is represented by the photon number state $|0\rangle|1\rangle$, and $|1\rangle$ is represented by the photon number state $|1\rangle|0\rangle$ - this encoding is also called the dual-rail encoding [26]. The only resources available are single photon sources, passive linear optics elements (such as beam splitters and phase shifters), and photon detectors. In our model, the only source of hardware imperfection is the efficiency of the photon detectors, that is, the photon sources and passive linear optics elements are assumed to be perfect. We also assume that classical computation and control are delay and error free, and that all sources of failure - teleportation and measurement failures - are statistically independent.

While single qubit operations can be efficiently performed using only phase shifters and beam-splitters [3] - and therefore can be considered error free in our model - two qubit operations require state post-selection through measurement of ancillary modes. If the desired measurement is not obtained, the operation may or may not have been applied properly, but if the desired measurement is obtained, the proper operation is guaranteed to have been applied. In Ref. 1 a probabilistic sign shift gate, which performs the operation $\mathrm{NS}_{-}=|0\rangle\langle 0|+| 1\rangle\langle 1|-| 2\rangle\langle 2|$ on number states of a given mode, is described. This gate succeeds with probability $\frac{1}{4}$. A probabilistic entangling gate CSIGN, which performs the operation $|00\rangle\langle 00|+| 01\rangle\langle 01|+| 10\rangle\langle 10|-| 11\rangle\langle 11|$ on two qubits, can be constructed by using two NS - gates along with two beam splitters, but no extra ancillae or measurements. Since both NS_ applications must succeed, the overall probability of 


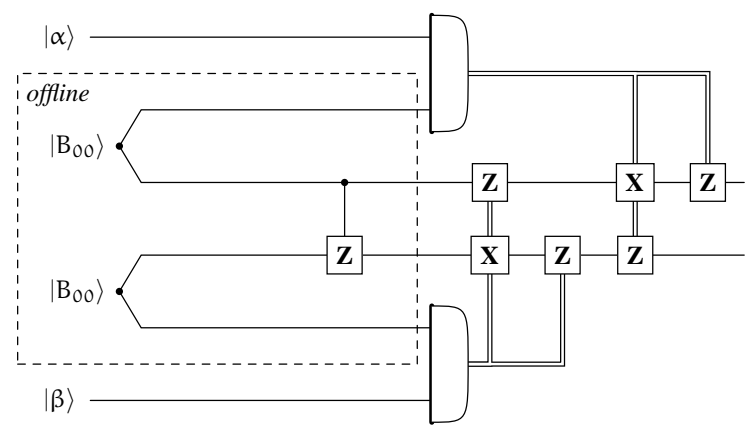

FIG. 1: Gate teleportation protocol for a CSIGN. The resource state is $\left|B_{00}\right\rangle=\frac{|00\rangle+|11\rangle}{\sqrt{2}}$ and the two qubit measurement is a Bell basis measurement. The double lines indicate operations conditioned on the outcome of the measurement.

success for a CSIGN is $\frac{1}{16}$. In order to make this construction scalable, one has to use gate teleportation [4], which turns the gate construction problem into a state preparation problem with the advantage that such states can be prepared off line without a priori knowledge of the inputs to the gate. The idea of gate teleportation is to use the conjugation relations of certain gates to modify the resource state and the correction operations of the teleportation protocol, so that these gates can be applied implicitly during the teleportation, in a manner similar to the protocol for the CSIGN gate illustrated in Figure 1 Strictly speaking, the CSIGN construction is based on the teleportation of some of the modes constituting the qubits that must interact, and thus the operations are not general unitaries but instead restricted to linear optics operations, single photon sources, and post-selection based on single photon detection of certain modes.

Even though the direct CSIGN construction has a small (but non-zero) probability of success, it is always known when the teleportation succeeds, and the resource state of the teleportation is independent of the inputs to the operation so one can make many attempts to produce such a resource state before performing the teleportation. Alternatively, one may make many attempts in parallel, and select one that succeeds. While without teleportation the number of attempts needed for error free quantum computation grows exponentially for circuits constructed with the probabilistic CSIGN gates, with the gate teleportation it grows linearly [1, 2]. Since the CSIGN along with all single qubit unitaries form a universal set for quantum computation [5], this gives an efficient construction for a universal quantum computer using only linear optics elements.

Teleportation in linear optics cannot be performed deterministically [6], but it can be performed with probability arbitrarily close to one at the cost of higher complexity for the teleportation protocol [1]. Once again, as in the probabilistic CSIGN construction, one can determine whether the protocol succeeded through the measurement of ancillary modes, so possible failures are always flagged by the measurement outcomes. Since it is not clear how to increase the probability of success of the direct CSIGN construction, and bounds for possible probabilities of success are predicted to be significantly smaller than one [7], this gate teleportation scheme is highly necessary. We say that the probability of these intrinsic teleportation failures occurring is $\epsilon_{\text {ideal }}$, since such failures occur even when considering ideal photon detectors (i.e. with perfect efficiency) and linear optics elements.

What makes the teleportation scheme highly attractive as well is exactly what the failure entails. When one of the teleportations involved in the CSIGN fails (but no detectors fail), the output is equivalent to a successful teleportation followed by a measurement in the $\mathbf{Z}$ eigenbasis - that is, we can view it as a successful gate application followed by a $\mathbf{Z}$ measurement of one of the qubits independently with probability $\epsilon_{\text {ideal }}$, and such an event is automatically flagged by the outcome of the measurement of the ancilla modes. This is what we call the ideal hardware error model, and it has been shown to be very benign, with an error threshold arbitrarily close to one [2, 8].

Hence teleportation failures under this model mean that one of the projectors into the eigenbasis of $\mathbf{Z}$ has been applied. Exactly which projector was applied to the teleported qubit depends on the outcome of the measurement of the ancillae. Formally, the projectors are $\mathbf{Z}_{+}=\frac{1}{2}(\mathbb{1}+\mathbf{Z})$ and $\mathbf{Z}_{-}=\frac{1}{2}(\mathbb{1}-\mathbf{Z})$. Since the location of these measurements is flagged by post-selection, this type of failure is a form of erasure - an error of known location.

\section{LOSSY HARDWARE ERROR MODEL}

One of the largest technical hurdles in the implementation of these probabilistic gates is the fact that single photon detectors are notoriously difficult to build. While for some wavelength very high efficiency can be obtained, the rate at which false photon detections are signaled, the so called dark counts, is unacceptably high [9]. Dark counts are particularly troublesome for the gates proposed in Ref.1 because they could cause photon loss to go undetected, as well as causing incorrect post-selection of states. Recent proposals of photon detectors based on phase transitions in superconductors have very low dark count rates, although little attention has been given to optimizing the efficiency of these detectors. We are interested in finding the minimum efficiency necessary for these detectors, given dark counts stay negligible, in order to be able to perform useful quantum computation [27].

Modifications to the gate teleportation protocol that allow for the detection of photon loss at the detectors are known [1]. This protocol can differentiate between the teleportation failures due to the limitations of linear optics (leaving the error model due to such failures intact) and the failures due to photon loss at the detectors - when this occurs, the corrupted qubit is replaced with a fresh qubit in a known state. We take the detection of a single physical qubit to fail with probability $\delta$, and the overall probability of the CSIGN gate teleportation failing due to photon loss at the detectors to be $\epsilon_{\text {loss. }}$. The form of $\epsilon_{\text {loss }}$ as a function of $\delta$ depends on the choice of protocol used - Ref.1 describes a family of protocols - and for the purposes of this paper, we will take them to be independent parameters. Once again, any possible error due to these types of failure is always flagged by the gate construction, which significantly simplifies error correction since it is known a priori where the 
failures have occurred.

Consider the error model due only to photon loss at the detectors $\left(\epsilon_{\text {ideal }}=0\right)$ - such an error model is not physical, since a linear optics quantum computer will always have teleportation failures, but taking this limiting case simplifies the analysis significantly. If we consider a single physical qubit measurement where photon loss occurred, it is clear that all information about the qubit is lost. We can model this loss of information by the full erasure superoperator

$$
\mathbb{E}(\rho)=\frac{1}{2} \mathbb{1}=\frac{1}{4}(\rho+\mathbf{X} \rho \mathbf{X}+\mathbf{Z} \rho \mathbf{Z}+\mathbf{Y} \rho \mathbf{Y})
$$

However, because of the dual-rail encoding, it is always clear which physical qubit measurement failed in such a manner since at the lowest level of encoding qubit measurement consist of measuring the two constituent modes, and thus only one of the detectors may click. If a photon is not detected on either mode, a measurement failure has occurred, and we may replace the qubit with a fresh qubit in a known state. This is fundamentally different from the depolarizing channel [10] where there is no a priori knowledge of the position of the errors - one may think of this superoperator as the depolarizing channel superoperator conditioned on perfect information about which qubits were randomized.

It is clear that the full erasure does not commute with gate teleportation, otherwise we could in principle transfer some of the information from the control qubit of a CSIGN to the target even though all information was lost in the failed teleportation of the control qubit. We take a worst case approach, and assume that any photon loss during one of the teleportations yields total information loss of the qubit being teleported, and that the error model for the other qubit associated with the same gate teleportation in which the photon was lost is determined by which correction might have been needed to be applied. In the particular case of the linear optics proposal, the error model is symmetric: if we disregard the classical correlation between the errors of the outputs of the CSIGN, photon loss in one of the teleportations translates to a full erasure of the qubit being teleported and a $\mathbf{Z}$ erasure of the other qubit involved in the gate operation. This is because in both cases the correction operation from one teleportation to the other is a $\mathbf{Z}$ gate, and if it is not known whether such gate was supposed to be applied because of the photon loss, we have the superoperator

$$
\mathbb{Z}(\rho)=\frac{1}{2}(\rho+\mathbf{Z} \rho \mathbf{Z}),
$$

which is what we call a $\mathbf{Z}$ erasure. Note that this superoperator can be interpreted as an unintentional $\mathbf{Z}$ measurement of unknown outcome, since

$$
\mathbf{Z}_{+} \rho \mathbf{Z}_{+}+\mathbf{Z}_{-} \rho \mathbf{Z}_{-}=\frac{1}{2}(\rho+\mathbf{Z} \rho \mathbf{Z})=\mathbb{Z}(\rho)
$$

and thus this is fundamentally different from the phase erasure channel [10], since it provides the added a priori knowledge of when corruption has occurred or not. As mentioned before, we say that there is a probability $\epsilon_{\text {loss }}$ that photon loss occurs in one of the teleportations in the CSIGN implementation, which entails a full erasure of the qubit being teleported, and a $\mathbf{Z}$ erasure of the qubit to which it was coupled through the CSIGN application. The correlation between the erasures on these two qubits is ignored for our calculations, but could be exploited to obtain better thresholds.

In summary, all single qubit operations are taken to be error free, and the CSIGN is taken to introduce at each output qubit either $\mathbf{Z}$ measurements, full erasures, or $\mathbf{Z}$ erasures with finite probability. For simplicity, we consider the different error models independently, that is, we calculate the threshold for the case where only $\mathbf{Z}$ measurements occur (where the hardware is ideal, with $\epsilon_{\text {loss }}=0$ and $\delta=0$, but teleportation is imperfect, i.e. $\epsilon_{\text {ideal }} \neq 0$ ), and we calculate the threshold where only full and $\mathbf{Z}$ erasures occur (that is, where the teleportation protocol is perfect, $\epsilon_{\text {ideal }}=0$, but the detectors are not, so $\delta \neq 0$ and $\epsilon_{\text {loss }} \neq 0$ ).

A collection of single qubit erasures is referred to as an erasure pattern, and the weight of the erasure pattern is the number of qubits that have been affected by an erasure, regardless of the type of erasure.

\section{FAULT-TOLERANCE AND ERROR CORRECTION}

The quantum error correction code considered here to protect the data from the error model in question is a $[[7,1,3]]$ self-orthogonal, doubly-even CSS code [11, 12] with stabilizer generators

$$
\begin{aligned}
& \mathbf{M}_{1}=\mathbf{X} \otimes \mathbf{X} \otimes \mathbf{X} \otimes \mathbf{X} \otimes \mathbb{1} \otimes \mathbb{1} \otimes \mathbb{1} \\
& \mathbf{M}_{\mathbf{2}}=\mathbf{X} \otimes \mathbf{X} \otimes \mathbb{1} \otimes \mathbb{1} \otimes \mathbf{X} \otimes \mathbf{X} \otimes \mathbb{1} \\
& \mathbf{M}_{3}=\mathbf{X} \otimes \mathbb{1} \otimes \mathbf{X} \otimes \mathbb{1} \otimes \mathbf{X} \otimes \mathbb{1} \otimes \mathbf{X} \\
& \mathbf{M}_{\mathbf{4}}=\mathbf{Z} \otimes \mathbf{Z} \otimes \mathbf{Z} \otimes \mathbf{Z} \otimes \mathbb{1} \otimes \mathbb{1} \otimes \mathbb{1} \\
& \mathbf{M}_{5}=\mathbf{Z} \otimes \mathbf{Z} \otimes \mathbb{1} \otimes \mathbb{1} \otimes \mathbf{Z} \otimes \mathbf{Z} \otimes \mathbb{1} \\
& \mathbf{M}_{\mathbf{6}}=\mathbf{Z} \otimes \mathbb{1} \otimes \mathbf{Z} \otimes \mathbb{1} \otimes \mathbf{Z} \otimes \mathbb{1} \otimes \mathbf{Z} .
\end{aligned}
$$

Self-orthogonal CSS codes are particularly suited for the error model considered here because they yield simple constructions of fault-tolerant encoded Clifford group operations. Although the Clifford group is not a universal set of quantum operations, it is well known how to extend it in order to obtain a universal set fault-tolerantly [13, 14], as well as how this affects the threshold value [15]. In the case of the error models considered here, the threshold is unaffected since we require that computation be performed only on error free states - that is, error correction is performed until an uncorrectable error occurs, causing the computation to be aborted, or until the data is error free, at which point the computation may continue.

Recall that the Clifford group consists of all operations that preserve the Pauli group under conjugation, and this group is generated by the CSIGN gate, the Hadamard gate $\mathbf{H}=$ $\frac{1}{\sqrt{2}}\left(\begin{array}{cc}1 & 1 \\ 1 & -1\end{array}\right)$, and the phase gate $\mathbf{P}=\left(\begin{array}{ll}1 & 0 \\ 0 & i\end{array}\right)$. The CSIGN can be implemented transversally by qubitwise CSIGNs between two encoded qubits. Note that CSS codes have a transversal encoded CSIGN. If in addition $\mathbf{H}$ has to be transversal, then the CSS code has to be constructed from a single, self-orthogonal classical code. Moreover, if $\mathbf{P}$ also has to 
be transversal, then all codewords in this classical code must have doubly-even weight. Since the 7-qubit code considered is both doubly-even and constructed from a self-orthogonal classical code, the Hadamard and phase gates can be implemented using only single qubit operations qubitwise, and therefore are error free under the models considered here. In order to obtain a universal gate set, we can add a non-Clifford gate such as the $\pi / 8$ gate, which has a known fault-tolerant construction [14]. We will make use of the properties of doubly-even CSS codes for the error correction circuits studied in SectionVI

\section{ENCODED ERROR MODEL}

Error models consisting of erasures yield particularly simple encoded error models because it is always known when an error is unrecoverable. In such a case one can simply take the code block to be an encoded erasure at the next level of encoding. In general, an uncorrectable failure is not an encoded failure, and it requires further processing to make it an encoded failure, as will be discussed below. What remains to be determined is what kind of encoded failure results.

In the case of ideal hardware, all erasures are $\mathbf{Z}$ measurements of known outcomes. Turning to the 7 qubit code described above, we have that all weight one and two erasure patterns are correctable. Of the 35 possible weight three erasure patterns, 28 are correctable. The remaining 7 weight three patterns, along with all other patterns with higher weight [28], can be identified with an encoded $\mathbf{Z}$ measurement, and are therefore uncorrectable failures. These collections of individual single qubit measurements are not, strictly speaking, equivalent to an encoded measurement, since they collapse the seven qubits into a state outside the code space. However, because the encoded $|\mathbf{0}\rangle$ and $|\mathbf{l}\rangle$ are superpositions over mutually exclusive sets of states, it is easy to infer which encoded state the measurement results correspond to, and then replace the qubits with a fresh encoded $|0\rangle$ or $|\mathbf{}\rangle$. This operation is taken to be error free, since we assume that state preparation can be attempted until no errors have occurred. Since all encoded failures are $\mathbf{Z}$ measurements, in the ideal hardware error model the break even condition between encoded $\mathbf{Z}$ measurements at the first level of encoding, denoted by $\mathbf{Z}^{(1)}$, and single qubit $\mathbf{Z}$ measurements, $\operatorname{Pr}\left(\mathbf{Z}^{(1)}\right.$ measurement $)=$ $\operatorname{Pr}(\mathbf{Z}$ measurement $)$, implies

$$
\epsilon_{\text {ideal }}^{(1)}=\epsilon_{\text {ideal }},
$$

where $\epsilon_{\text {ideal }}^{(1)}$ is the failure rate at the first level of encoding.

In the case of the lossy error model, because each qubit can suffer either $\mathbf{Z}$ erasures or full erasures, different uncorrectable errors on the 7 qubit code will lead to different encoded errors. For simplicity we can take all encoded failures to be encoded full erasures, which in the first level of encoding we denote $\mathbb{E}^{(1)}$, so that the break even condition on the probabilities $\operatorname{Pr}\left(\mathbb{E}^{(1)}\right)=\operatorname{Pr}(\mathbb{E})$ implies

$$
\epsilon_{\text {loss }}^{(1)}=\frac{1}{2} \epsilon_{\text {loss }}
$$

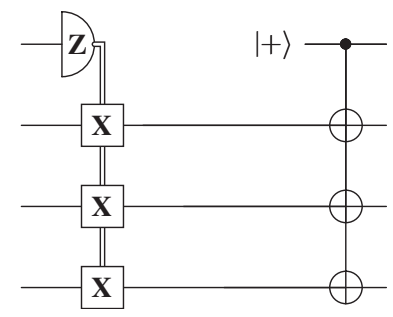

FIG. 2: A circuit for correcting a single $\mathbf{Z}$ measurement in the top qubit as long as the remaining 3 qubits are erasure-free. The four qubits are assumed to be part of a block encoded with the 7 qubit code. The state $|+\rangle$ is the +1 eigenvalue eigenvector of $\mathbf{X}$. The double lines indicate control based on the outcome of the measurement.

since only half of the lossy error model failures are full erasures, where $\epsilon_{\text {loss }}^{(1)}$ is the failure rate at the first level of encoding. The resulting threshold is at most as high as the real threshold, considering the different kinds of encoded erasures that are simpler to correct, but should be lower in general. A more detailed analysis can be made [17, 18], and exact probabilities distributions for the different kinds of erasures can be calculated using the same technique used to calculate the threshold in Section VII but the simplifying assumption made here is enough to match the prediction in Ref. 2.

In reality we would like to consider an error model that takes both these sources of error into account. Section IV describes how each of the different kinds of erasures are corrected, and demonstrates the progressively higher cost of correcting a $\mathbf{Z}$ measurement, a $\mathbf{Z}$ erasure and a full erasure. Given this fact, for the error correction code chosen here, and for the error correction technique used here, the threshold for an error model consisting of both types of failures is bounded above by the ideal error model threshold, and below by the lossy error model threshold.

\section{ERROR CORRECTION CIRCUITS}

In general, in order to correct errors by using a stabilizer code, one simply needs to measure the stabilizer generators and infer the most probable error that occurred and apply the correction. In the case of erasure errors, the knowledge of which qubits have been affected by the error superoperator greatly reduces the number of stabilizer operators that need to be measured. This is because we need only measure stabilizer operators that act non-trivially on the qubits affected by the error superoperators, and this greatly reduces the probability of introducing more errors into the data.

This procedure can be further optimized by incorporating the syndrome measurement and correction into a single step, as first described in Ref. 2 for the case of $\mathbf{Z}$ measurement correction codes.

The fact that the 7 qubit code employed here is based on a classical doubly-even code, allows us to consider the 4 qubit subsystem in the support of any given stabilizer operator. Since this is a CSS code, we can focus on stabilizer operators that are made up of tensor products of $\mathbf{X s}$ and identities, and 


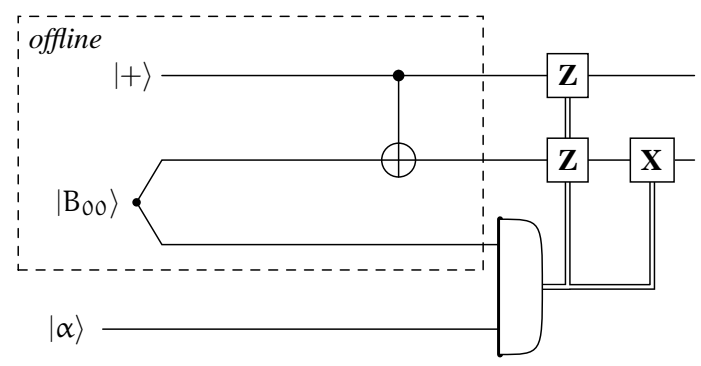

FIG. 3: Performing a CNOT between $|+\rangle$ and an arbitrary state $|\alpha\rangle$ through teleportation.

stabilizer operators that are made up of tensor products of $\mathbf{Z s}$ and identities.

Since we are only interested in correcting erasures of weight up to 3 , we can consider a single qubit that has undergone some erasure, along with three qubits that are still intact. We choose stabilizer operators that act non-trivially on all of these four qubits and trivially on all other qubits - this is always possible in the 7 qubit code. Considering only this 4 qubit subsystem, the stabilizer operators in question are [29]

$$
\begin{aligned}
& \mathbf{M}_{1}^{\prime}=\mathbf{X} \otimes \mathbf{X} \otimes \mathbf{X} \otimes \mathbf{X} \\
& \mathbf{M}_{2}^{\prime}=\mathbf{Z} \otimes \mathbf{Z} \otimes \mathbf{Z} \otimes \mathbf{Z} .
\end{aligned}
$$

The usual approach is to measure these two operators faulttolerantly in order to determine what kind of Pauli correction needs to be applied to the erased qubit. If we consider $\mathbf{Z}$ erasures, we need only measure $\mathbf{M}_{1}^{\prime}$. Alternatively, one can simply use the circuit depicted in Figure 2 (without loss of generality, we consider the corrupted qubit to be the first qubit of the four) [30]. As demonstrated before, a code that can correct $\mathbf{Z}$ erasures can also correct $\mathbf{Z}$ measurements at known locations, since a $\mathbf{Z}$ erasure can be given by a $\mathbf{Z}$ measurement of unknown outcome. In this case, we do not need to perform the explicit measurement in the $\mathbf{Z}$ eigenbasis since we already have that information, but the rest of the circuit remains as in Figure 2 - thus there is an added cost of a possible measurement failure when correcting $\mathbf{Z}$ erasures. If the measurement does fail and the qubit is destroyed (as is the case when a photon is detected), one simply abandons the attempt at correcting the $\mathbf{Z}$ erasure, since it will then be replaced by a full erasure.

The circuit in Figure 2 is not fault-tolerant, as it stands. In order to make it fault-tolerant, one simply applies a modified teleportation protocol to the three error-free qubits [1, 8, 17]. In order to understand why this is fault-tolerant, consider the teleportation of the three intact qubits before applying the CNOTs in Figure 2 Since CNOT is a Clifford gate, we can apply the CNOTs to the Bell states needed for teleportation, and simply modify the recovery stage of the teleportation, in a manner similar to what was described in Figure 10 Note that it is unnecessary to teleport the control qubit, i.e. the qubit in the state $|+\rangle$, since it is a fixed resource state. Unlike the CS IGN gate construction, where photon modes are teleported, this is a teleportation of the qubits, and can be thought of in terms of the usual higher level gates such as CNOTs and Pauli

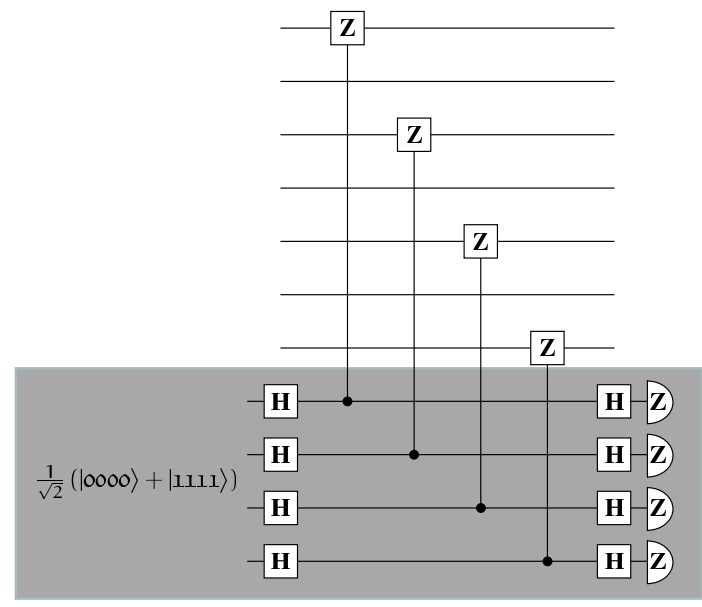

FIG. 4: Fault-tolerant measurement of stabilizer operator $\mathbf{Z} \otimes \mathbb{1} \otimes$ $\mathbf{Z} \otimes \mathbb{1} \otimes \mathbf{Z} \otimes \mathbb{1} \otimes \mathbf{Z}$ as proposed by Shor [13]. The qubits in the shaded region are ancillas, and the measurement of the operator is inferred from the measurement of the 4 ancilla qubits.

operators.

This procedure becomes clearer if we consider the teleportation of only one of the qubits, followed by the CNOT with the resource state $|+\rangle$, and propagate the CNOT backwards, as described in Figure 3 . If there is a failure in the Bell measurement of the qubits, it will only possible cause a $\mathbf{Z}$ erasure in the control bit, as well as a possible full erasure on the target bit. Since $\mathbf{Z}$ errors do not propagate from the control of the CNOT gate, the other two CNOTs can be performed fault tolerantly in parallel.

In summary, we simply need to measure the erased qubit in the $\mathbf{Z}$ eigenbasis, discard the erased bit, and apply the teleportation-based fault-tolerant version of Figure 2 to three erasure free qubits in the codeword and an extra qubit in the $|+\rangle$ state to replace the discarded one, and the $\mathbf{Z}$ erasure will be corrected. If any of the teleportations fail, the failure will affect only the qubit we are attempting to recover and the qubit that was being teleported.

The circuit in Figure 4 is used to measure stabilizer operators made of tensor products of $\mathbf{Z s}$ and $\mathbb{1} \mathrm{s}$, and thus it partially corrects full erasures, yielding a $\mathbf{Z}$ erasure if successful. The fault-tolerant version of the circuit in Figure 2 can be used to correct $\mathbf{Z}$ erasures as well as unintentional $\mathbf{Z}$ measurements. The strategy taken is to correct full erasures first, and once there are no more full erasures, to correct $\mathbf{Z}$ erasures and $\mathbf{Z}$ measurements. We do not claim that this strategy and circuits are optimal for error correction, but this choice simplifies the exact calculation of the threshold significantly.

\section{THRESHOLDS}

The threshold theorem [19, 20, 21] states that by concatenated coding - that is, the repeated encoding of a quantum state - one can perform quantum computation with arbitrarily small error efficiently as long as the error is below a certain 
threshold. We take this threshold to be the smallest probability of error such that the probability of an encoded failure $\epsilon^{(1)}$ is equal to the probability of a single unencoded qubit failure $\epsilon^{(0)} \equiv \epsilon$.

The encoded error rate can be calculated by tracking the probability of going from any given erasure pattern to any other erasure pattern during an attempt at error correction. This describes a Markov chain, and such a description can be made more compact by considering symmetries of the error correction code and of the error correction circuitry [17]. Erasure patterns can be grouped into equivalence classes defined by the error correcting code as well as the error correcting procedure, and we need only consider probabilities of going from one equivalence class to another - in the case of the 7 qubit code, we need to consider only 11 equivalence classes versus the 128 that would be necessary for a naive description of the Markov chain. It is straightforward to obtain the initial distribution of the different equivalence classes as well as the transition matrix of the Markov chain that describes the change in the distribution due to one error correction attempt. The distribution after multiple error correction attempts can be obtained by taking higher powers of the transition matrix and applying it to the initial distribution. Each of the non-trivial equivalence classes of erasure patterns can be associated with an encoded erasure, and therefore one can obtain the error distribution at any given encoding level. In some cases, there is additional processing and transitions between different equivalence classes associated with the mapping between the erasure pattern and an encoded erasure, since, for example the erasure operator $\mathbb{1} \otimes \mathbb{1} \otimes \mathbb{1} \otimes \mathbb{1} \otimes \mathbb{1} \otimes \mathbb{1} \otimes \mathbb{E}$ is not equivalent to any encoded operation. In order to account for such processing, another transition matrix would be required. Details of this procedure are discussed elsewhere [18]. In the case of the ideal hardware error model, all erasure patterns can be mapped directly to encode measurements by measuring all the qubits of a code block that is not erasure free. In the case of the lossy hardware model, we take a worst case approach and all erasure patterns at the end of the error correction procedure are taken to be an encoded full erasure, so there is no need for the more detailed analysis - we can simply replace the corrupted block of qubits with a block in a known fixed encoded state.

In the case of perfect hardware $(\delta=0)$, the error rate recursion relation is

$\epsilon_{\text {ideal }}^{(1)}=56 \epsilon_{\text {ideal }}^{3}+406 \epsilon_{\text {ideal }}^{4}+3878 \epsilon_{\text {ideal }}^{5}-129675 \epsilon_{\text {ideal }}^{6}+\cdots$,

which yields a threshold, for $\epsilon_{\text {ideal }}^{(1)}=\epsilon_{\text {ideal }}$, of approximately $\epsilon_{\text {ideal }}=0.115$. The Markov chain describing the error recovery procedure for this model is shown in Figure 5

In the case of lossy hardware with perfect teleportation, assuming that $\delta=\epsilon_{\text {loss }}$, the error rate recursion relation is

$\epsilon_{\text {loss }}^{(1)}=1050 \epsilon_{\text {loss }}^{3}+33173 \epsilon_{\text {loss }}^{4}-46242 \epsilon_{\text {loss }}^{5}-6861701 \epsilon_{\text {loss }}^{6}+\cdots$,

which yields a threshold, for $\epsilon_{\text {loss }}^{(1)}=\frac{1}{2} \epsilon_{\text {loss }}$, of approximately $\epsilon_{\text {loss }}=0.0178$. This threshold is only valid if it is identical to or smaller than the encoded measurement threshold. Because

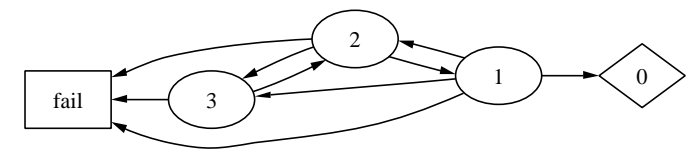

FIG. 5: The Markov chain describing the error recovery for the ideal hardware error model. The states are labeled by the weight of the correctable erasures, and the 'fail' corresponds to all patterns that are not correctable according to our procedure. Probabilites are omitted for readability.

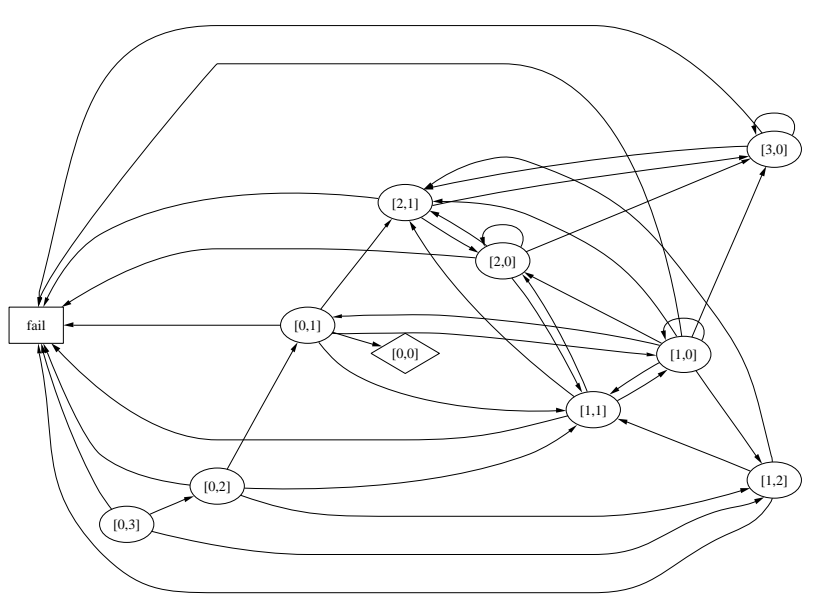

FIG. 6: The Markov chain describing the error recovery for the lossy hardware error model. The states are labeled $[m, n]$ according to the number of full erasures $m$ and the number of $\mathbf{Z}$ erasures $n$, and the 'fail' state corresponds to all patterns that are not correctable according to our procedure. Probabilites are omitted for readability.

of the structure of CSS codes, encoded basis states correspond to superpositions of elements of a coset of a linear classical code. In the case of the $[[7,1,3]]$ quantum code, the linear classical code is a $[7,4,3]$ code. Measurement failures can then be seen as classical erasures on this $[7,4,3]$ code, and ignoring the correctable classical erasure patterns of weight three and higher, the encoded failure rate for measurements is given by

$$
\delta^{(1)}=\sum_{i=3}^{7}\left(\begin{array}{l}
7 \\
i
\end{array}\right) \delta^{i}(1-\delta)^{7-i}
$$

which yields the benign error threshold $\delta=0.25$, validating the calculated threshold value $\epsilon_{\text {loss }}=0.0178$ under the assumption $\delta=\epsilon_{\text {loss }}$. This is a worst case assumption because the family of teleportation protocols described in Ref. 1 use an increasing number of detectors to increase the probability of success, and for the smallest such protocol the probability of photon loss is the same as the probability of photon loss for a single qubit measurement. The Markov chain for the lossy hardware error model is depicted in Figure 6 


\section{CONCLUSIONS}

Using the error correction techniques outline here, the error threshold for Clifford gates is found to be at least $0.0178<\epsilon<0.115$ (since $\epsilon_{\text {loss }}<\epsilon<\epsilon_{\text {ideal }}$ ), where $\epsilon$ is the probability that some type of erasure is introduced due to photon loss at the detectors or due to a teleportation failure.

The threshold values calculated here can be improved by using optimized stabilizer measurement techniques [22, 23], or by merging stabilizer measurement and error correction steps more aggressively [8, 24]. Figure 4] is a straightforward generalization of a technique used for a two qubit $\mathbf{Z}$ measurement error correcting code [2], but Knill showed that by merging all stabilizer measurements with error correction steps in modified teleportation protocols, significantly higher thresholds can be obtained [24].

The Markov chain description of the error correction procedure, discussed in more detail elsewhere [18], can be used with any of these techniques. This systematic approach to the calculation of the encoded error rates is particularly useful for practical applications of concatenated codes, since it is able to give the probability distribution of the encoded errors. The calculation to check the dependency of the distribution on parameters such as the number of error correction attempts or the number of concatenation levels is straightforward, and one could determine easily how many correction attempts or concatenation levels are necessary to obtain some target error rate.

\section{Acknowledgments}

M.S. would like to thank Michele Mosca and Raymond Laflamme for their support. This work was carried out while M.R. and C.Z. were at the Institute for Quantum Computing, University of Waterloo. This research was partially funded by NSERC, MITACS and ARDA.
[1] E. Knill, R. Laflamme, and G. Milburn, Nature 409, 46 (2001).

[2] E. Knill, R. Laflamme, and G. Milburn (2000), quant$\mathrm{ph} / 0006120$.

[3] M. Reck, A. Zeilinger, H. J. Bernstein, and P. Bertani, Physical Review Letters 73, 58 (1994).

[4] D. Gottesman and I. L. Chuang, Nature 402, 390 (1999).

[5] M. A. Nielsen and I. L. Chuang, Quantum Computation and Quantum Information (Cambridge University Press, 2000).

[6] N. Lütkenhaus, J. Calsamiglia, and K.-A. Suominen, Physical Review A 59, 3295 (1999).

[7] E. Knill (2003), quant-ph/0307015.

[8] E. Knill, in ITP Program on Quantum Information: Entanglement, Decoherence and Chaos ((online), 2001), pp. I-V, http://online.itp.ucsb.edu/online/qinfo01/.

[9] R. Sobolewski, A. Verevkin, G. N. Gol'tsman, A. Lipatov, and K. Wilsher, IEEE Transactions on Applied Superconductivity 13, 1151 (2003).

[10] C. Bennett, D. DiVincenzo, and J. Smolin, Physical Review Letters 78, 3217 (1997).

[11] A. R. Calderbank and P. W. Shor, Physical Review A 54, 1098 (1996).

[12] A. M. Steane, Proceedings of the Royal Society of London A 452, 2551 (1996).

[13] P. Shor, in 37th Symposium on Foundations of Computing, IEEE (IEEE Computer Society Press, 1996), pp. 56-65.

[14] X. Zhou, D. W. Leung, and I. L. Chuang, Physical Review A 62, 052316 (2000).

[15] D. Gottesman, Ph.D. thesis, California Institute of Technology (1997).

[16] D. Gottesman, Physical Review A 57, 127 (1998).
[17] M. Silva, Master's thesis, University of Waterloo (2004), quant$\mathrm{ph} / 0405112$.

[18] M. Silva and M. Rötteler, in preparation.

[19] D. Aharonov and M. Ben Or (1999), quant-ph/9906129.

[20] E. Knill, R. Laflamme, and W. Zurek (1996), quant$\mathrm{ph} / 9610011$.

[21] J. Preskill, Introduction to Quantum Computation (World Scientific, 1998), chap. Fault-tolerant quantum computation, quant-ph/9712048.

[22] A. M. Steane (2002), quant-ph/0202036.

[23] A. M. Steane and B. Ibson (2003), quant-ph/0311014.

[24] E. Knill, quant-ph/0312190.

[25] M. Grassl, T. Beth, and T. Pellizzari, Physical Review A 56, 33 (1997).

[26] In a photon number state, each ket represents an optical mode, and the number represents the number of photons occupying that mode.

[27] In order to consider dark count induced errors, one would need to look into a general error model, not an erasure error model.

[28] There are weight four patterns that are correctable, but we ignore them for simplicity, since the error recovery operation used here is not applicable to these patterns.

[29] Note that this is different from the 4 qubit erasure code described by Grassl and collaborators [25], because for any 4 qubits we consider, there will be other stabilizer operators in the 7 qubit code that are not generated by these two.

[30] To see how this circuit works, simply consider the action of the circuit on the states stabilized by (7). 